\title{
Asymmetric Epoxidation of Terminal Alkenes with Hydrogen Peroxide Catalyzed by Pentafluorophenyl Pt" Complexes
}

\author{
Marco Colladon, ${ }^{\dagger}$ Alessandro Scarso, ${ }^{\dagger}$ Paolo Sgarbossa, ${ }^{\ddagger}$ Rino A. Michelin ${ }^{\ddagger}$ and Giorgio Strukul ${ }^{*}$, \\ †Dipartimento di Chimica, Università Ca' Foscari di Venezia, Dorsoduro 2137, 30123, Venice, (Italy) \\ ${ }^{\ddagger}$ Dipartimento di Processi Chimici dell'Ingegneria, Università di Padova, Via F. Marzolo 9, 35131 Padova, (Italy)
}

Supporting Information Available: Full experimental procedures and characterization data for complexes $1 \mathrm{a}, 1 \mathrm{~b}$ and $1 \mathrm{~d}$.

\section{Experimental Section}

\section{General}

${ }^{1} \mathrm{H}$ NMR and ${ }^{31} \mathrm{P}\left\{{ }^{1} \mathrm{H}\right\}$ NMR spectra were recorded at $298 \mathrm{~K}$, unless otherwise stated, on a Bruker AVANCE 300 spectrometer operating at 300.15 and $121.50 \mathrm{MHz}$, respectively, while ${ }^{19} \mathrm{~F}\left\{{ }^{1} \mathrm{H}\right\}$ were recorded on a Bruker AC200 operating at $188.25 \mathrm{MHz}$. $\delta$ values in ppm are relative to $\mathrm{SiMe}_{4}, 85 \% \mathrm{H}_{3} \mathrm{PO}_{4}$ and $\mathrm{CFCl}_{3}$. GC measurement were taken on a Hewlett-Packard 5890A gas chromatograph equipped with a FID detector (gas carrier $\mathrm{He}$ ). All reactions except propene oxidation were monitored on a $25 \mathrm{~m} \mathrm{HP-5}$ capillary column I.D. $0.32 \mathrm{~mm}$, film 0.25 m. Product yields were calculated using the internal standard method. Identification of products was made with GLC, GC-MS and NMR by comparison with authentic samples. Elemental analyses were performed by the Department of Analytical, Inorganic and Organometallic Chemistry of Università di Padova. Enantiomeric excess was determined as reported in Table S1.

Table S1. Enantiomeric excess and absolute configuration assignment of terminal epoxides.

ee
determination $\begin{gathered}\text { Abs. Config. } \\ \text { assignment }\end{gathered}$




\begin{tabular}{rll}
\hline & $A$ & 1 \\
$A$ & $A$ & 1 \\
\hline
\end{tabular}

A) e.e. determined by ${ }^{1} \mathrm{H}-\mathrm{NMR}$ integration with the chiral shift reagent Europium tris-[3(heptafluoropropylhydroxymethylene)-(+)-camphorate] ( $\mathrm{Eu}(\mathrm{hfc}) 3)$ at RT. B) e.e. determined by CSP-GC on a $25 \mathrm{~m} \beta$-cyclodex column I.D. $0.25 \mathrm{~mm}$, film $0.25 \_\mathrm{m}, \mathrm{T}_{\text {inj }}=250^{\circ} \mathrm{C} \mathrm{T}_{\text {det }}=300^{\circ} \mathrm{C}$, carrier He $1 \mathrm{~mL} / \mathrm{min}, 45^{\circ} \mathrm{C} \times 30$ min then $5^{\circ} \mathrm{C} / \mathrm{min}$ up to $200^{\circ} \mathrm{C}$; $\mathrm{t}_{\mathrm{S}} 18.1 \mathrm{~min}, \mathrm{t}_{R} 19.3 \mathrm{~min}$. C) e.e. determined by chiral HPLC analysis on a Chiracel-ODH column, $100 \%$ hexane, $0.6 \mathrm{~mL} / \mathrm{min}, \mathrm{t}_{s} 34.9 \mathrm{~min}, \mathrm{t}_{R} 37.1 \mathrm{~min}$. D) e.e. determined by chiral HPLC analysis on a Chiracel-ODH column, 97\% hexane-3\% 2-propanol, $1 \mathrm{~mL} / \mathrm{min}, \mathrm{t}_{s} 7.7 \mathrm{~min}, \mathrm{t}_{R} 8.3 \mathrm{~min}$. E) e.e. determined by chiral HPLC analysis on a Chiracel-ODH column, $97 \%$ hexane-3\% 2-propanol, $1 \mathrm{~mL} / \mathrm{min}$, $\mathrm{t}_{s} 20.0 \mathrm{~min}, \mathrm{t}_{R} 22.3 \mathrm{~min}$. F) e.e. determined by chiral HPLC analysis on a Chiracel-ODH column, $97 \%$ hexane-3\% 2-propanol, $\left.1 \mathrm{~mL} / \mathrm{min}, \mathrm{t}_{s} 33.0 \mathrm{~min}, \mathrm{t}_{R} 38.1 \mathrm{~min} . \mathrm{G}\right)$ e.e. determined by chiral HPLC analysis on a Chiracel-ODH column, $99.3 \%$ hexane-0.7\% 2-propanol, $1 \mathrm{~mL} / \mathrm{min}, \mathrm{t}_{s} 16.4 \mathrm{~min}, \mathrm{t}_{R} 17.3 \mathrm{~min}$. H) Absolute configuration determined by addition of enantiomerically pure $(R)-(+)$-propene oxide to the enantioenriched epoxides in the presence of $\left(\mathrm{Eu}(\mathrm{hfc})_{3}\right)$ at $\mathrm{RT}$. I) Absolute configuration assigned from comparison to the deshielding effect in the presence of $\left(\mathrm{Eu}(\mathrm{hfc})_{3}\right)$ at $\mathrm{RT}$ observed for propene oxide. J) Absolute configuration assigned from the GC elution order in $\beta$-cyclodex column. K) The absolute configuration was determined by comparing the sign of the measured optical rotations with literature data. ${ }^{1} \mathrm{~L}$ ) Absolute configuration determined by HPLC elution order on a Chiracel-ODH column compared to the other aromatic substrates above reported. M) The absolute configuration was determined by comparing the sign of the measured optical rotations with literature data. ${ }^{2} \mathrm{~N}$ ) The absolute configuration was determined by comparing the sign of the measured optical rotations with literature data. ${ }^{3}$

\section{Materials}

Hydrogen peroxide $(35 \%$ and $60 \%$ Aldrich) as well as all the alkene substrates listed above are commercial products (Aldrich or Fluka) and were used without further purification. The chiral complex [(S,S)chiraphosPt $\left(\mathrm{C}_{6} \mathrm{~F}_{5}\right)\left(\mathrm{H}_{2} \mathrm{O}\right)$ ]OTf 1c was prepared following the procedure reported in the literature, ${ }^{4}$ while complexes 1a, 1b, 1d were prepared with similar procedures.

\section{Materials for $\mathrm{Pt}^{\prime \prime}$ complexes Synthesis}

All synthetic steps were carried out under dinitrogen atmosphere using standard Schlenck techniques. Solvents were dried and purified according to standard methods. ${ }^{5}$ The diphosphines (+)-1,2-bis((2R,5R)-2,5di-i-propylphospholano)benzene, 98+\% ( $R, R$ )-i-Pr-DUPHOS 2a, (1S,1'S,2R,2'R)-(+)-1,1'-Di-t-butyl-[2,2']diphospholane, 97\% (S,S,R,R)-TANGPHOS 2 b , (2R,3R)-(-)-2,3-bis(diphenylphosphino)-bicyclo[2.2.1]hept-5ene, min. $95 \%(R, R)$-NORPHOS $\mathbf{2 d}$ were commercially available from STREM chemicals and were used as purchased.

$\left[\mathrm{Pt}\left(\mathrm{C}_{6} \mathrm{~F}_{5}\right)(\mu-\mathrm{Cl})(\text { tht })\right]_{2}{ }^{6}$ was synthesized following procedures reported in the literature. Hydrogen peroxide (35\% Fluka), $\mathrm{MgCl}^{-} \mathrm{Pr}, \mathrm{AgBF}_{4}, \mathrm{AgOTf}$, were commercial products and used without purification. The chiral complex $\left[(S, S)\right.$-chiraphosPt $\left.\left(\mathrm{C}_{6} \mathrm{~F}_{5}\right)\left(\mathrm{H}_{2} \mathrm{O}\right)\right] \mathrm{OTf}$ 1c was prepared following the procedure reported in the literature. ${ }^{4}$ 


\section{Oxidation Reactions}

These were carried out in a $2 \mathrm{~mL}$ vial equipped with a screw-capped silicone septum to allow sampling. Stirring was performed by a Teflon-coated bar driven externally by a magnetic stirrer $(700 \mathrm{rpm})$. Constant temperature was maintained by water or alcohol circulation through an external jacket connected with a thermostat. The concentration of the commercial $35 \% \mathrm{H}_{2} \mathrm{O}_{2}$ solution was checked iodometrically prior to use. Typically, the proper amount of catalyst $1 \mathrm{a}-\mathrm{d}(0.016 \mathrm{mmol})$ was places in the vial, followed by the solvent ( 1 $\mathrm{mL}$ ) and the internal standard ( $n$-decane), then the vial was thermostatted. After stirring for $10 \mathrm{~min}$, the substrate $(0.83 \mathrm{mmol})$ was added and the mixture stirred for $10 \mathrm{~min}$. To this, hydrogen peroxide was added in one portion $(0.83 \mathrm{mmol})$ and time was started. All reactions (except propene oxidation which was followed by ${ }^{1} \mathrm{H}-\mathrm{NMR}$ analysis) were monitored with GC by direct injection of samples taken periodically from the reaction mixtures with a microsyringe. Prior quenching of the samples by adding an excess of $\mathrm{LiCl}$ was found to be unnecessary, except for propene AE.

\section{Synthetic Procedures for 1a, 1b and 1d}

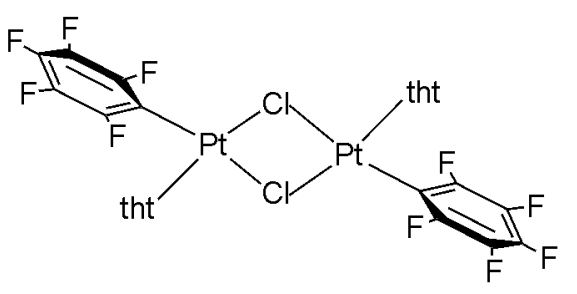

tht=tetrahydrothiophene

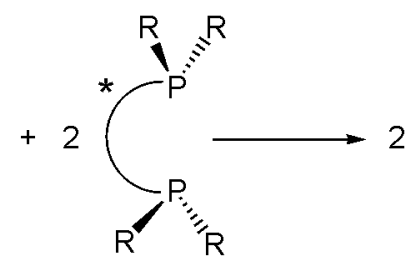

2a-d

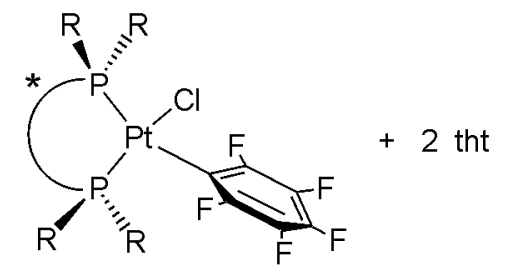

3a-d

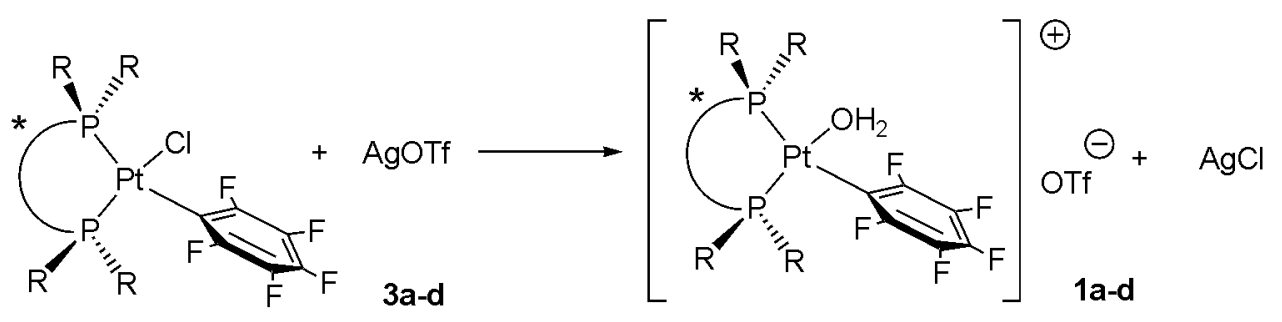<smiles>CC(C)[C@@H]1CC[C@@H](C(C)C)P1c1ccccc1P1[C@H](C(C)C)CC[C@@H]1C(C)C</smiles>

$(R, R, R, R)-2 \mathrm{a}$

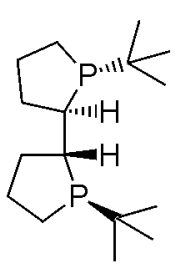

$(S, S, R, R)-2 b$<smiles>CC(P(c1ccccc1)c1ccccc1)P(c1ccccc1)c1ccccc1</smiles>

$(S, S)-2 c$<smiles>C1=CC2CC1[C@H](P(c1ccccc1)c1ccccc1)[C@@H]2P(c1ccccc1)c1ccccc1</smiles>

$(R, R)-2 \mathrm{~d}$

$\left[\mathrm{PtCl}\left(\mathrm{C}_{6} \mathrm{~F}_{5}\right)\left((\boldsymbol{R}, \boldsymbol{R})\right.\right.$-i-Pr-DUPHOS] 3a. To a solution of $\left[\mathrm{Pt}(\mu-\mathrm{Cl})\left(\mathrm{C}_{6} \mathrm{~F}_{5}\right)(\text { tht })\right]_{2}(0.12 \mathrm{~g}, 0.12 \mathrm{mmol})$ in acetone $(30 \mathrm{~mL})$ at room temperature were added $0.10 \mathrm{~g}(0.24 \mathrm{mmol})$ of $(R, R)-i$-Pr-DUPHOS. The solution was stirred for 2 hours then concentrated and treated with $n$-pentane giving a white solid, which was filtered off and dried under vacuum. Yield $0.067 \mathrm{~g}, 35 \%$. Anal. Calc. for $\mathrm{C}_{32} \mathrm{H}_{44} \mathrm{ClF}_{5} \mathrm{P}_{2} \mathrm{Pt}$ : C, 47.09; $\mathrm{H}, 5.43 \%$; Found: $\mathrm{C}$, 
47.11; $\mathrm{H}, 5.40 ; \%$. ${ }^{1} \mathrm{H}$ NMR ( $\delta, \mathrm{CD}_{2} \mathrm{Cl}_{2}$ ): 7.86-7.50 (m, Ar), 3.37-2.70 (m, alkyl), 2.63-1.64 (m, alkyl), 1.18 (d, $6.1 \mathrm{~Hz}, i \operatorname{Pr}), 1.08$ (d, $6.4 \mathrm{~Hz}, i \operatorname{Pr}), 0.97$ (d, $6.4 \mathrm{~Hz}, i \operatorname{Pr}), 0.89$ (d, $6.4 \mathrm{~Hz}, i \operatorname{Pr}), 0.79$ (d, $6.7 \mathrm{~Hz}, i \operatorname{Pr}), 0.72(\mathrm{~d}, 6.4$ $\mathrm{Hz}, i \mathrm{Pr}), 0.70(\mathrm{~d}, 6.4 \mathrm{~Hz}, i \mathrm{Pr}), 0.62(\mathrm{~d}, 6.7 \mathrm{~Hz}, i \mathrm{Pr}) ;{ }^{31} \mathrm{P}\left\{{ }^{1} \mathrm{H}\right\} \mathrm{NMR}\left(\delta, \mathrm{CD}_{2} \mathrm{Cl}_{2}\right): 54.42\left(\mathrm{~d}, \mathrm{P}_{\mathrm{O} \text {-trans }},{ }^{1} \mathrm{~J}_{\mathrm{Pt}-\mathrm{P}}=\right.$ $\left.3732 \mathrm{~Hz},{ }^{2} \mathrm{~J}_{\mathrm{P}-\mathrm{P}}=5.9 \mathrm{~Hz}\right) ; 60.02\left(\mathrm{~m}, \mathrm{P}_{\mathrm{C}-\text { trans }},{ }^{1} \mathrm{~J}_{\mathrm{Pt}-\mathrm{P}}=2232 \mathrm{~Hz}\right) ;{ }^{19} \mathrm{~F}\left\{{ }^{1} \mathrm{H}\right\} \mathrm{NMR}\left(\delta, \mathrm{CD}_{2} \mathrm{Cl}_{2}\right):-116.20,-121.23(\mathrm{~m}$, $o-F),-162.45\left(t, p-F,{ }^{3} J_{F-F}=20.3 \mathrm{~Hz}\right),-164.15,-164.97(m, m-F)$.

[PtCl$\left.\left(\mathrm{C}_{6} \mathrm{~F}_{5}\right)((\boldsymbol{S}, \boldsymbol{S}, \boldsymbol{R}, \boldsymbol{R})-\mathrm{TANGPHOS})\right]$ 3b. This compound was prepared using a similar procedure to that described above for complex 3 a starting from $\left[\mathrm{Pt}(\mu-\mathrm{Cl})\left(\mathrm{C}_{6} \mathrm{~F}_{5}\right)(\text { tht })\right]_{2}(0.165 \mathrm{~g}, 0.17 \mathrm{mmol})$ in chloroform (30 $\mathrm{mL})$ and $0.10 \mathrm{~g}(0.35 \mathrm{mmol})$ of $(\mathrm{S}, \mathrm{S}, R, R)$-TANGPHOS. Yield $0.148 \mathrm{~g}, 63 \%$. Anal. Calc. for $\mathrm{C}_{22} \mathrm{H}_{32} \mathrm{ClF}_{5} \mathrm{P}_{2} \mathrm{Pt}$ : $\mathrm{C}, 38.63 ; \mathrm{H}, 4.72 \%$. Found: $\mathrm{C}, 38.72 ; \mathrm{H}, 4.60 \% .{ }^{1} \mathrm{H}$ NMR $\left(\delta, \mathrm{CD}_{2} \mathrm{Cl}_{2}\right): 2.75-1.86$ (m, alkyl), 1.80-1.55 (m, alkyl), $1.41(\mathrm{~d}, 14.3 \mathrm{~Hz}, t \mathrm{Bu}), 1.04(\mathrm{~d}, 14.3 \mathrm{~Hz}, t \mathrm{Bu}) ;{ }^{31} \mathrm{P}\left\{{ }^{1} \mathrm{H}\right\} \mathrm{NMR}\left(\delta, \mathrm{CD}_{2} \mathrm{Cl}_{2}\right): 86.30$ (d, $\mathrm{P}_{\mathrm{O} \text {-trans }},{ }^{1} \mathrm{~J}_{\mathrm{Pt}-\mathrm{P}}=3670$ $\left.\mathrm{Hz},{ }^{2} \mathrm{~J}_{\mathrm{P}-\mathrm{P}}=3.1 \mathrm{~Hz}\right) ; 96.17\left(\mathrm{~m}, \mathrm{P}_{\mathrm{C}-\text { trans }},{ }^{1} \mathrm{~J}_{\mathrm{Pt}-\mathrm{P}}=2228 \mathrm{~Hz}\right) ;{ }^{19} \mathrm{~F}\left\{{ }^{1} \mathrm{H}\right\} \mathrm{NMR}\left(\delta, \mathrm{CD}_{2} \mathrm{Cl}_{2}\right):-117.42,-122.11(\mathrm{~m}, \mathrm{o}-\mathrm{F})$, $162.91\left(\mathrm{t}, p-\mathrm{F},{ }^{3} \mathrm{~J}_{\mathrm{F}-\mathrm{F}}=19.1 \mathrm{~Hz}\right),-163.80,-165.67(\mathrm{~m}, m-\mathrm{F})$.

$\left[\mathrm{PtCl}\left(\mathrm{C}_{6} \mathrm{~F}_{5}\right)((\boldsymbol{R}, \boldsymbol{R})\right.$-NORPHOS $\left.)\right]$ 3d. This compound was prepared using a similar procedure to that described above for complex 3a starting from $\left[\mathrm{Pt}(\mu-\mathrm{Cl})\left(\mathrm{C}_{6} \mathrm{~F}_{5}\right)(\text { tht })\right]_{2}(0.15 \mathrm{~g}, 0.15 \mathrm{mmol})$ in chloroform $(30 \mathrm{~mL})$ and $0.15 \mathrm{~g}(0.31 \mathrm{mmol})$ of $(R, R)-\mathrm{NORPHOS}$. Yield $0.220 \mathrm{~g}, 83 \%$. Anal. Calc. for $\mathrm{C}_{37} \mathrm{H}_{28} \mathrm{ClF}_{5} \mathrm{P}_{2} \mathrm{Pt}$ : C, 51.67; $\mathrm{H}, 3.28 \%$; Found: $\mathrm{C}, 51.88 ; \mathrm{H}, 3.09 \%$. ${ }^{1} \mathrm{H}$ NMR $\left(\delta, \mathrm{CD}_{2} \mathrm{Cl}_{2}\right)$ : 8.24-7.68 (m, Ar), 7.65-7.37 (m, Ar), 7.36-7.22 (m, Ar), 7.15-6.95 (m, Ar), 6.23 (dd, J $3.3 \mathrm{~Hz}, 5.2 \mathrm{~Hz}$, aryl), 6.11 (dd, J $3.3 \mathrm{~Hz}, 5.2 \mathrm{~Hz}$, aryl), 5.39 (m, aryl), 3.50-3.21 (m, alkyl), 3.16 (s, alkyl), 3.04 (s, alkyl), 2.87 (s, alkyl), 2.70 (s, alkyl), 2.35 (m, alkyl), 1.05-0.70 (m, alkyl); ${ }^{31} \mathrm{P}\left\{{ }^{1} \mathrm{H}\right\} \operatorname{NMR}\left(\delta, \mathrm{CD}_{2} \mathrm{Cl}_{2}\right): 12.52\left(\mathrm{~d}, \mathrm{P}_{\mathrm{O}-\text { trans }},{ }^{1} \mathrm{~J}_{\mathrm{Pt}-\mathrm{P}}=3784 \mathrm{~Hz},{ }^{2} \mathrm{~J}_{\mathrm{P}-\mathrm{P}}=9.8 \mathrm{~Hz}\right), 11.47\left(\mathrm{~d}, \mathrm{P}_{\mathrm{O} \text {-trans }},{ }^{1} \mathrm{~J}_{\mathrm{Pt}-\mathrm{P}}=\right.$ $\left.3790 \mathrm{~Hz},{ }^{2} \mathrm{~J}_{\mathrm{P}-\mathrm{P}}=12.2 \mathrm{~Hz}\right), 9.60\left(\mathrm{~m}, \mathrm{P}_{\mathrm{C}-\text { trans }},{ }^{1} \mathrm{~J}_{\mathrm{Pt}-\mathrm{P}}=2286 \mathrm{~Hz}\right), 8.54\left(\mathrm{~m}, \mathrm{P}_{\mathrm{C}-\text { trans }},{ }^{1} \mathrm{~J}_{\mathrm{Pt}-\mathrm{P}}=2294 \mathrm{~Hz}\right) ;{ }^{19} \mathrm{~F}\left\{{ }^{1} \mathrm{H}\right\} \mathrm{NMR}$ $\left(\delta, \mathrm{CD}_{2} \mathrm{Cl}_{2}\right):-120.14,-121.34(\mathrm{~m}, \mathrm{o}-\mathrm{F}),-163.48\left(\mathrm{t}, p-\mathrm{F},{ }^{3} \mathrm{~J}_{\mathrm{F}-\mathrm{F}}=20.3 \mathrm{~Hz}\right),-165.09,-166.44(\mathrm{~m}, m-\mathrm{F})$.

[Pt $\left(\mathrm{C}_{6} \mathrm{~F}_{5}\right)\left(\mathrm{H}_{2} \mathrm{O}\right)\left((\boldsymbol{R}, \boldsymbol{R})\right.$-i-Pr-DUPHOS)][OTf] 1a. To a solution of $\left[\mathrm{PtCl}\left(\mathrm{C}_{6} \mathrm{~F}_{5}\right)((R, R)-\mathrm{i}-\mathrm{Pr}\right.$-DUPHOS $\left.)\right](0.066 \mathrm{~g}$, $0.08 \mathrm{mmol})$ in dichloromethane $(25 \mathrm{~mL})$ were added $0.5 \mathrm{~mL}$ of an acetone solution of AgOTf $(0.083 \mathrm{mmol})$. The suspension was stirred for an hour then the solid $\mathrm{AgCl}$ was filtered off and the solution was concentrated. Upon treatment with $n$-pentane a pale yellow solid was obtained, filtered off and dried under vacuum. Yield 0.051g, 66\%. Anal. Calc. for $\mathrm{C}_{33} \mathrm{H}_{46} \mathrm{~F}_{8} \mathrm{O}_{4} \mathrm{P}_{2} \mathrm{PtS}$ : $\mathrm{C}, 41.82 ; \mathrm{H}, 4.89 \%$; Found: $\mathrm{C}, 41.70 ; \mathrm{H}$, 5.01\%. ${ }^{1} \mathrm{H}$ NMR $\left(\delta, \mathrm{CD}_{2} \mathrm{Cl}_{2}\right):$ 7.90-7.61 (m, Ar), 3.09-2.87 (m, alkyl), 2.66-1.44 (m, alkyl), 1.25 (d, $\left.6.6 \mathrm{~Hz}, \mathrm{Pr}\right)$, 1.16 (d, $6.6 \mathrm{~Hz}, i \operatorname{Pr}), 1.04$ (d, $6.6 \mathrm{~Hz}, i \operatorname{Pr}), 0.92(\mathrm{~d}, 6.6 \mathrm{~Hz}, i \operatorname{Pr}), 0.85$ (d, $6.6 \mathrm{~Hz}, i \operatorname{Pr}), 0.76$ (d, $6.3 \mathrm{~Hz}, i \operatorname{Pr}), 0.74$ (d, $6.3 \mathrm{~Hz}, i \mathrm{Pr}), 0.66(\mathrm{~d}, 6.6 \mathrm{~Hz}, i \mathrm{Pr}) ;{ }^{31} \mathrm{P}\left\{{ }^{1} \mathrm{H}\right\} \mathrm{NMR}\left(\delta, \mathrm{CD}_{2} \mathrm{Cl}_{2}\right): 44.48\left(\mathrm{~d}, \mathrm{P}_{\mathrm{O}-\text { trans }},{ }^{1} \mathrm{~J}_{\mathrm{Pt}-\mathrm{P}}=4348 \mathrm{~Hz},{ }^{2} \mathrm{~J}_{\mathrm{P}-\mathrm{P}}=6.3\right.$ $\mathrm{Hz}$ ); 64.89 (m, $\mathrm{P}_{\mathrm{C}-\text { trans }},{ }^{1} \mathrm{~J}_{\mathrm{Pt}-\mathrm{P}}=2282 \mathrm{~Hz}$ ); ${ }^{19} \mathrm{~F}\left\{{ }^{1} \mathrm{H}\right\} \mathrm{NMR}\left(\delta, \mathrm{CD}_{2} \mathrm{Cl}_{2}\right):-79.34$ (s, OTf), -120.91, -121.77 (m, o-F), $-161.30(\mathrm{~m}, p-\mathrm{F}),-164.41,-165.02(\mathrm{~m}, m-\mathrm{F})$.

[Pt $\left(\mathrm{C}_{6} \mathrm{~F}_{5}\right)\left(\mathrm{H}_{2} \mathrm{O}\right)((\mathrm{S}, \mathrm{S}, \boldsymbol{R}, \boldsymbol{R})$-TANGPHOS $\left.)\right][\mathrm{OTf}]$ 1b. This compound was prepared using a similar procedure to that described above for complex 1a starting from $\left[\mathrm{PtCl}\left(\mathrm{C}_{6} \mathrm{~F}_{5}\right)((\mathrm{S}, S, R, R)-\mathrm{TANGPHOS})\right](0.14 \mathrm{~g}, 0.20 \mathrm{mmol})$. Yield $0.12 \mathrm{~g}, 74 \%$. Anal. Calc. for $\mathrm{C}_{23} \mathrm{H}_{34} \mathrm{~F}_{8} \mathrm{O}_{4} \mathrm{P}_{2} \mathrm{PtS}$ : C, 33.87; $\mathrm{H}, 4.20 \%$; Found: $\mathrm{C}, 33.72 ; \mathrm{H}, 4.33 \%$. ${ }^{1} \mathrm{H}$ $\operatorname{NMR}\left(\delta, \mathrm{CD}_{2} \mathrm{Cl}_{2}\right)$ : 2.69-1.98 (m, alkyl), 1.90-1.55 (m, alkyl), 1.38 (d, 14.8 Hz, tBu), 1.14 (d, $\left.14.8 \mathrm{~Hz}, t \mathrm{Bu}\right) ;{ }^{31} \mathrm{P}$ $\left\{{ }^{1} \mathrm{H}\right\} \operatorname{NMR}\left(\delta, \mathrm{CD}_{2} \mathrm{Cl}_{2}\right): 77.15\left(\mathrm{~d}, \mathrm{P}_{\mathrm{O}-\text { trans }},{ }^{1} \mathrm{~J}_{\mathrm{Pt}-\mathrm{P}}=4204 \mathrm{~Hz},{ }^{2} \mathrm{~J}_{\mathrm{P}-\mathrm{P}}=3.1 \mathrm{~Hz}\right) ; 99.76\left(\mathrm{~m}, \mathrm{P}_{\mathrm{C}-\text { trans }},{ }^{1} \mathrm{~J}_{\mathrm{Pt}-\mathrm{P}}=2256 \mathrm{~Hz}\right)$; 
${ }^{19} \mathrm{~F}\left\{{ }^{1} \mathrm{H}\right\} \operatorname{NMR}\left(\delta, \mathrm{CD}_{2} \mathrm{Cl}_{2}\right):-79.36(\mathrm{~s}, \mathrm{OTf}),-116.75,-122.63(\mathrm{~m}, \mathrm{o}-\mathrm{F}),-159.62(\mathrm{~m}, p-\mathrm{F}),-162.45,-164.12(\mathrm{~m}$, $m-\mathrm{F})$.

$\left[\mathrm{Pt}\left(\mathrm{C}_{6} \mathrm{~F}_{5}\right)\left(\mathrm{H}_{2} \mathrm{O}\right)((\boldsymbol{R}, \boldsymbol{R})-\mathrm{NORPHOS})\right][\mathrm{OTf}]$ 1d. This compound was prepared using a similar procedure to that described above for complex 1a starting from [PtCl$\left.\left(\mathrm{C}_{6} \mathrm{~F}_{5}\right)((R, R)-\mathrm{NORPHOS})\right](0.202 \mathrm{~g}, 0.23 \mathrm{mmol})$. Yield $0.218 \mathrm{~g}, 93 \%$. Anal. Calc. for $\mathrm{C}_{38} \mathrm{H}_{30} \mathrm{~F}_{8} \mathrm{O}_{4} \mathrm{P}_{2} \mathrm{PtS}$ : C, 46.02; $\mathrm{H}, 3.05 \%$; Found: $\mathrm{C}, 45.89 ; \mathrm{H}, 3.16 \%$. ${ }^{1} \mathrm{H}$ NMR $(\delta$, $\mathrm{CD}_{2} \mathrm{Cl}_{2}$ ): 8.18-7.91 (m, Ar), 7.74-7.44 (m, Ar), 7.37-7.28 (m, Ar), 7.16-7.02 (m, Ar), 6.23 (dd, J $3.3 \mathrm{~Hz}, 5.2 \mathrm{~Hz}$, aryl), 6.12 (dd, J $3.3 \mathrm{~Hz}, 5.2 \mathrm{~Hz}$, aryl), 5.47 (dd, J $2.7 \mathrm{~Hz}, 5.5 \mathrm{~Hz}$, aryl), 5.36 (dd, J $2.7 \mathrm{~Hz}, 5.5 \mathrm{~Hz}$, aryl), 3.44-3.25 (m, alkyl), 3.21 (s, alkyl), 3.04 (s, alkyl), 2.93 (s, alkyl), 2.91-278 (m, alkyl), 2.69 (s, alkyl), 2.34-2.11 (m, alkyl), 1.90-1.57 (m, alkyl), 0.95 (d, $9.1 \mathrm{~Hz}$,alkyl), 0.90 (d, $6.6 \mathrm{~Hz}$, alkyl), 0.87 (d, $6.6 \mathrm{~Hz}$, alkyl), 0.77 (d, $9.1 \mathrm{~Hz}$, alkyl); ${ }^{31} \mathrm{P}\left\{{ }^{1} \mathrm{H}\right\} \operatorname{NMR}\left(\delta, \mathrm{CD}_{2} \mathrm{Cl}_{2}\right): 5.84\left(\mathrm{~d}, \mathrm{P}_{\mathrm{O} \text {-trans }},{ }^{1} \mathrm{~J}_{\mathrm{Pt}-\mathrm{P}}=4410 \mathrm{~Hz},{ }^{2} \mathrm{~J}_{\mathrm{P}-\mathrm{P}}=13.5 \mathrm{~Hz}\right), 4.84\left(\mathrm{~d}, \mathrm{P}_{\mathrm{O} \text {-trans }}\right.$, $\left.{ }^{1} \mathrm{~J}_{\mathrm{Pt}-\mathrm{P}}=4410 \mathrm{~Hz},{ }^{2} \mathrm{~J}_{\mathrm{P}-\mathrm{P}}=13.5 \mathrm{~Hz}\right), 16.63\left(\mathrm{~m}, \mathrm{P}_{\mathrm{C}-\text { trans }},{ }^{1} \mathrm{~J}_{\mathrm{Pt}-\mathrm{P}}=2388 \mathrm{~Hz}\right), 14.31\left(\mathrm{~m}, \mathrm{P}_{\mathrm{C}-\text { trans }},{ }^{1} \mathrm{~J}_{\mathrm{Pt}-\mathrm{P}}=2388 \mathrm{~Hz}\right) ;{ }^{19} \mathrm{~F}$ $\left\{{ }^{1} \mathrm{H}\right\} \operatorname{NMR}\left(\delta, \mathrm{CD}_{2} \mathrm{Cl}_{2}\right):-79.23$ (s, OTf), -120.66, -121.92 (m, o-F), -160.87 (m, p-F), -164.49, -165.05 (m, $m-$ $\mathrm{F})$.

\section{References}

(1) A. Córdova; H. Sundén; A. Bøgevig; M. Johansson; F. Himo Chem. Eur. J. 2004, 10, 3673-3684.

(2) E. Pinard; A. Alanine; A. Bourson; B. Buettelmann; R. Gill; M.-P. Heitz; G. Jaeschke; V. Mutel; G. Trube; R. Wyler; Bioorg. Med. Chem. Lett. 2001, 11, 2173 - 2176.

(3) O. W. Gooding; R.P. Bansal Synth. Commun. 1995, 25, 1155-1166.

(4) E. Pizzo; P. Sgarbossa; A. Scarso; R.A. Michelin; G. Strukul Organometallics, 2006, 25, 3056-3062.

(5) D.D. Perrin; W.L.F. Armarego, Purification of Laboratory Chemicals, $3^{\text {rd }}$ Edition, Pergamon Press, 1988.

(6)(a) Uson, R.; Fornies, J.; Espinet, P.; Alfranca, G. Synth. React.Inorg. Met.-Org. Chem. 1980, 10, 597. (b) Uson, R.; Fornies, J.; Martinez, F.; Tomas, M. J. Chem. Soc., Dalton Trans. 1980, 888. 